\title{
Automatic IVUS Segmentation of Atherosclerotic Plaque with Stop \& Go Snake
}

\author{
Ellen Brunenberg ${ }^{1}$, Oriol Pujol ${ }^{2}$, Bart ter Haar Romeny ${ }^{1}$, and Petia Radeva ${ }^{2}$ \\ ${ }^{1}$ Department of Biomedical Engineering, Eindhoven University of Technology, \\ P.O.Box 513, 5600 MB Eindhoven, the Netherlands \\ e.j.l.brunenberg@student.tue.nl, b.m.terhaarromeny@tue.nl \\ ${ }^{2}$ Computer Vision Center, Universitat Autònoma de Barcelona, Edifici O, \\ Campus UAB, 08193 Bellaterra (Cerdanyola), Barcelona, Spain \\ oriol@cvc.uab.es, petia@cvc.uab.es
}

\begin{abstract}
Since the upturn of intravascular ultrasound (IVUS) as an imaging technique for the coronary artery system, much research has been done to simplify the complicated analysis of the resulting images. In this study, an attempt to develop an automatic tissue characterization algorithm for IVUS images was done. The first step was the extraction of texture features. The resulting feature space was used for classification, constructing a likelihood map to represent different coronary plaques. The information in this map was organized using a recently developed [1] geodesic snake formulation, the so-called Stop \& Go snake. The novelty of our study lies in this last step, as it was the first time to apply the Stop \& Go snake to segment IVUS images.
\end{abstract}

\section{Introduction}

During the last decade, intravascular ultrasound has overtaken angiography as state-of-the-art visualization technique of atherosclerotic disease in coronary arteries. The most important property that determines the occurrence and outcome of acute coronary syndrome is the vulnerability, as opposed to the occlusion. Therefore coronary angiography was not the best method for risk determination, as it only displays a shadow of the lumen. Conversely, IVUS images show the morphological and histological properties of a cross-section of the artery. Different tissue types, such as soft plaque, fibrous plaque, and calcium, can be distinguished. Most significant in a vulnerable plaque is the presence of a large soft core with a thin fibrous cap. Although heavily calcified plaques seem to be more stable than non-calcified plaques, the amount of calcium is an indicator of the overall plaque burden, and as such, the degree of calcification will correlate with the overall risk of plaque rupture in the coronary arterial tree.

Despite the good vulnerability determination, IVUS has the disadvantage that manual analysis of the huge amount of images is difficult, subjective, and timeconsuming. Therefore, there is an increasing interest in the development of automatic tissue characterization algorithms for IVUS images. However, this is a challenging problem, as the image quality is poor due to noise, imaging artifacts and shadowing. A lot of research on this question has been done using 
extraction of texture features to characterize plaque tissues or determine plaque borders [234, recently in combination with classification techniques like AdaBoost 5. Deformable models are another extensively used technique to retrieve the intima and adventitia boundaries 6 6 78. Nevertheless, they are not commonly applied to segment different tissue types.

In this study, a new geodesic snake formulation, the so-called Stop \& Go snake [1, is employed to find the soft plaque and calcification regions in atherosclerotic plaques. This snake uses likelihood maps to decouple regularity and convergence, thus controlling the role of the curvature in a better way. To ensure convergence, the external force definition is split into an attractive and a repulsive vector field. We used this new snake in a traditional pattern recognition pipeline: first, the extraction of texture features, namely local binary patterns, co-occurrence matrices, and Gabor filters, was performed on the images. The second stage consists of classification using AdaBoost, addressing soft and fibrous plaque, and calcium. The confidence rate map obtained was used as a likelihood map for the Stop \& Go snake. The next section describes the feature extraction and classification. Section 3 discusses the fundamentals of the Stop and Go snake formulation and the use of likelihood maps. Section 4 gives the experimental results and section 5 concludes this paper.

\section{Feature Extraction and Classification}

The complexity of IVUS images can be reduced using feature extraction. Because pixel-based gray level-only methods are not sufficient to differentiate between the complicated structures, texture features are used. Examples of the latter discussed in previous studies are co-occurrence matrices and fractal analysis [2], run-length measures and radial profile [3], local binary patterns [4, derivatives of Gaussian, wavelets, Gabor filters, and cumulative moments 77. Because Pujol et al. achieved best results on IVUS tissue characterization using local binary patterns, co-occurrence matrices, and Gabor filters [4], our study was performed using these three sets of features.

After feature extraction, the feature space is used as input for an AdaBoost classifier. The idea behind boosting methods is that many simple, weak classifiers together can form a strong classification algorithm with many practical advantages [9]. AdaBoost is easy to implement and fast. However, before both feature extraction and classification can be performed, the images have to be preprocessed. The objective of this step is twofold: first, some of the images' marks and artifacts are removed to avoid their influence on feature extraction and classification. Furthermore, the image in Cartesian coordinates is converted into polar coordinates, to prevent biases from rotation-variant feature extractors.

\subsection{Feature Extractors}

With the preprocessed image as input, feature extractors usually give a high-dimensional feature vector as response. The Local Binary Pattern (LBP) 
operator, introduced by Ojala et al. [10], detects uniform local binary patterns within circularly symmetric neighborhoods of $P$ members with radius $R$.

The second feature operator, the gray-level co-occurrence matrix (COOC), provides a statistical tool for extraction of second-order texture information from images [11. The actual feature space is generated by the extraction of six measures from the co-occurrence matrices, namely energy, entropy, inverse difference moment, shade, inertia and promenance [7].

The last feature operators used for this study are the Gabor filters, originating from the multi-channel filtering theory. A Gabor filter consists of a sinusoidal, modulated in amplitude by a Gaussian envelope. By rotation of the coordinate system, filters at different orientations can be obtained. For practical purposes, the angles $\theta=\left\{0^{\circ}, 45^{\circ}, 90^{\circ}, 135^{\circ}\right\}$ suffice again.

\subsection{AdaBoost Classification}

The normalized feature data were classified using our AdaBoost algorithm, with decision stumps or one-level decision trees as weak classifiers. Let the training set contain $N$ samples, consisting of a $d$-dimensional feature vector $\boldsymbol{x}_{i}(i=1, \ldots, N)$ and a class label $y_{i}$. Decision stumps threshold these training data using only one out of the $d$ features, assigning a class label based on majority voting [12].

During boosting, such a weak classifier is called repeatedly in a series of rounds $t=1, \ldots, T$. The principle of this algorithm is to maintain a distribution of weights assigned to the training samples, denoted by $D_{t}(i)$. Initially, the samples are classified using equally set weights. In the next round, the weights for incorrectly classified samples are increased, while those for correct observations are reduced. This has the effect that the algorithm focuses on samples misclassified in the previous round, i.e. the difficult samples in the data set [9].

\section{Stop \& Go Snake}

In a traditional pattern recognition pipeline, classifiers like AdaBoost are used to find regions of interest after feature extraction. Subsequently, a deformable model can organize the obtained image information.

\subsection{Traditional Geodesic Deformable Models}

Geodesic active contours have a formulation based on curve evolution and level sets 13. A big advantage of this is that it can deal with topological changes during snake evolution. This evolution should find the curve of minimum length in a Riemannian surface with a measure depending on the image gradient. It follows that the snake $\Gamma$ evolves according to:

$$
\frac{\delta \Gamma}{\delta t}=\left(\boldsymbol{g} \cdot \boldsymbol{\kappa}+V_{0}-\langle\nabla \boldsymbol{g}, \boldsymbol{n}\rangle\right) \cdot \boldsymbol{n}
$$

with $\boldsymbol{g}=1 /\left(1+|\nabla I|^{2}\right)$ and $\boldsymbol{\kappa}$ the curvature of $\Gamma, \boldsymbol{n}$ its inward unit normal and $\langle$,$\rangle the scalar product of two vectors. It can be seen that the curvature$ 
has a double role, defining the motion of the curve at zero gradient regions, but also serving as a curve regularizing term, ensuring continuity. This has the disadvantage that it slows down the numeric scheme because it is a second-order term. Furthermore, it complicates snake convergence into concave areas.

\subsection{Stop \& Go Formulation}

To overcome the problems regarding convergence and regularity, a new definition where these terms are decoupled was introduced by Pujol et al. 11. In this socalled Stop \& Go snake, the curvature term does not interfere in the convergence process. The desired decoupling can be achieved using characteristic functions of the region of interest $R$ :

$$
I(x, y)= \begin{cases}1 & \text { if }(x, y) \in R, \\ 0 & \text { otherwise }\end{cases}
$$

By removing the influence of the curvature, any global vector field properly defining the target contour as its equilibrium ensures convergence. Such a vector field can be split into an exterior attractive field (the Go term) and an inner repulsive one (the Stop term), of which the sum cancels on the curve of interest. The separately defined Go and Stop terms are glued together by means of the characteristic function mentioned above.

If the evolving curve is outside the region of interest $R$, the Go term corresponds to an area minimization process restricted to the outside of $R$. The Go field ensures an inward motion to $R$, comparable to a balloon force. Because there is only need for a Stop field in the neighborhood of the desired contour, this term can be defined by the outward gradient $\boldsymbol{g}$ locally defining the contours of $R$. The Stop and Go snake evolution is then given by:

$$
\frac{\delta \Gamma}{\delta t}=\underbrace{\langle I \cdot \nabla \boldsymbol{g}, \boldsymbol{n}\rangle \boldsymbol{n}}_{\text {Stop }}+\underbrace{V_{0} \cdot(1-I) \cdot \boldsymbol{n}}_{\text {Go }}+\underbrace{\alpha \kappa \breve{I} \boldsymbol{n}}_{\text {Reg. term }} .
$$

The last term ensures smoothness and continuity. Because these conditions are only required in the final steps of the snake evolution, a restrictive mask $\breve{I}$ is used to define their scope, here $\breve{I}=G_{\sigma} * I$, with $G_{\sigma}$ a Gaussian filter with standard deviation $\sigma$. In spite of the resemblance of (3) to that of 'traditional' geodesic snakes, the role of the curvature is different here. It is now only a regularizing term weighted by $\alpha$. Furthermore, it does not trouble the convergence anymore because it only influences the last steps of the evolution.

\subsection{Likelihood Maps}

Unfortunately, for practical applications, there are no characteristic functions that define the regions of interest available, so an alternative function to generate the decoupling is needed. A likelihood map represents the probability of each pixel to belong to the object of interest. In general, a likelihood map only needs 
to fulfill the requirement that the object of interest is given as a local extremum. Examples of results that can be used as likelihood maps are the image's direct response to feature extractors or the outcome of the classifier. The latter is not very suitable, because it has very strong edges that cause the snake to follow simply the contours of this classification map. This is often not the optimal result and besides, there are easier and faster ways to find those edges. In this study, we propose to use the classifier's confidence rates as likelihood maps. These confidence rates can easily be extracted from the AdaBoost classification.

The normalized (between 0 and 1) version of the likelihood map $\breve{L}$ will be used in the Stop \& Go snake. Then, the only question remaining is the definition of the Stop term, $\breve{L} \cdot \nabla \boldsymbol{g}$. Pujol et al. [1] proposed to base this term on the likelihood map, defining it as $\nabla(1-\breve{L})$. All this leads to:

$$
\frac{\delta \Gamma}{\delta t}=\alpha \kappa \breve{L} \cdot \boldsymbol{n}+\beta\langle\nabla(1-\breve{L}), \boldsymbol{n}\rangle \cdot \boldsymbol{n}+V_{0}(1-\breve{L}) \cdot \boldsymbol{n},
$$

with $\alpha$ weighting the curvature's role and $\beta$ influencing the curve's smoothness.

\section{Results}

The IVUS images used in this study images were acquired by a last generation IVUS scanner (Galaxy, Boston Scientific). Compared to the previous ones, this scanner provides much better contrast and higher resolution, leading to a more expressed texture appearance of all intravascular structures. In this context, the analysis of these images is even more difficult compared to the previous generation images, justifying the texture analysis.

To be able to evaluate the results of the classifier and the snake correctly, IVUS images segmented manually by cardiologists from the Hospital Universitari "Germans Trias i Pujol" in Badalona, Spain, were used as a 'ground truth'. Furthermore, classifiers like AdaBoost, that perform supervised learning, need a set of training samples. In this study, a set of about 13,000 points was selected manually from the cardiologists' segmentation.

\subsection{Results of AdaBoost Classification}

For 30 IVUS images, two classifications were performed using 10 rounds of boosting, one separating fibrous plaque and calcium from soft plaque, and one distinguishing fibrous plaque from calcium. In the classified images shown in column $\mathrm{C}$ of Figs. 1 and 2 it can be seen that calcium and fibrous plaque are classified reasonably well, but that soft plaque apparently is difficult to segment. These observations are confirmed when looking at the confusion matrix in Table1 The majority of soft plaque, fibrous plaque and calcium points are classified correctly, although soft plaque is still repeatedly classified as fibrous. The percentage of points that are assigned correctly is $75.82 \%$. 

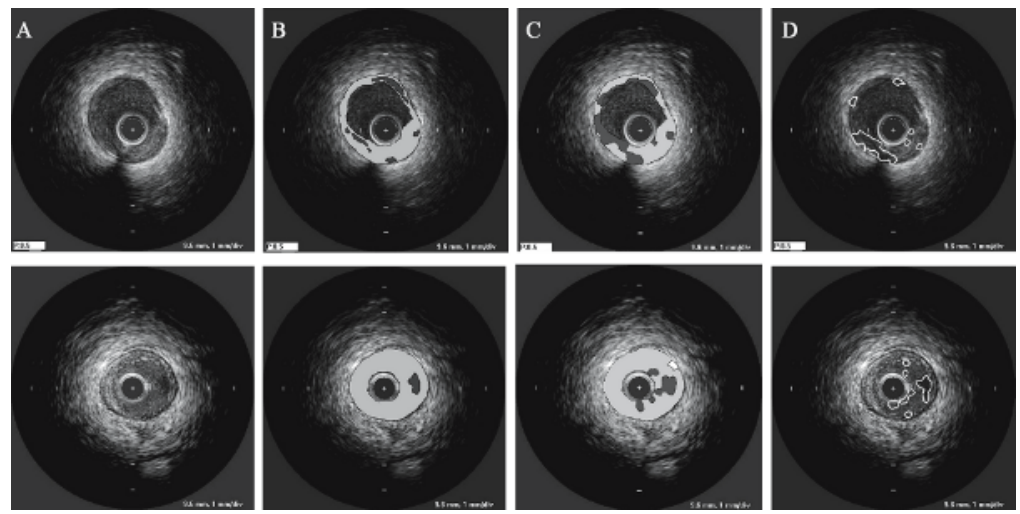

Fig. 1. Classification and snake results for two IVUS images with presence of soft plaque. Column A: Original images. B: Images segmented by cardiologist. C: AdaBoost classification map. D: Stop \& Go snake result. Legend for columns B and C: Dark gray represents soft plaque, light gray fibrous plaque and white calcification.
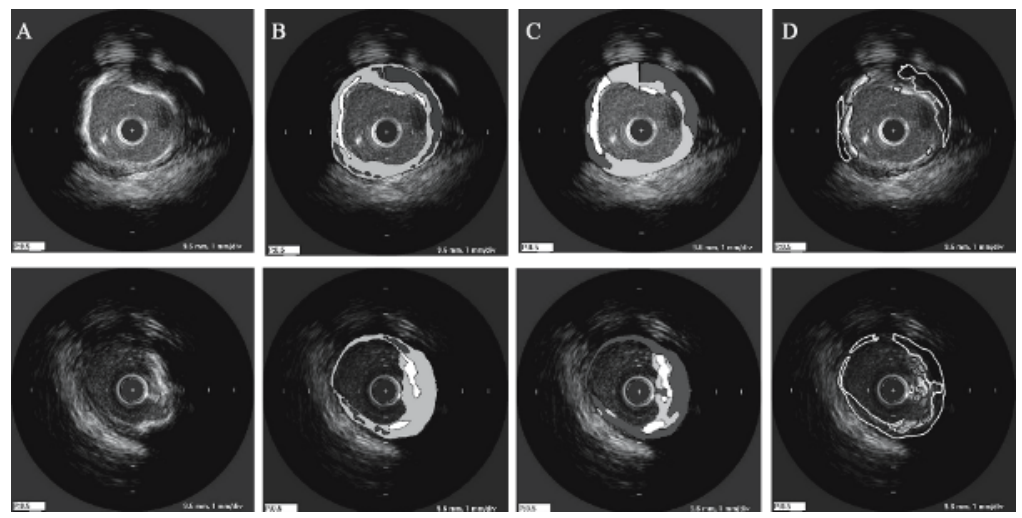

Fig. 2. Classification and snake results for two IVUS images with presence of soft plaque and calcium. Column A: Original images. B: Images segmented by cardiologist. C: AdaBoost classification map. D: Stop \& Go snake result. Legend for column D: White lines represent soft plaque, black ones calcification.

Table 1. Confusion matrix (in percents) for boosted decision stumps classification

\begin{tabular}{|l|l|l|l|l|}
\hline & $\begin{array}{l}\text { Labeled as soft } \\
\text { plaque }\end{array}$ & $\begin{array}{l}\text { Labeled as fibrous } \\
\text { plaque }\end{array}$ & $\begin{array}{l}\text { Labeled as cal- } \\
\text { cium }\end{array}$ & Totals \\
\hline $\begin{array}{l}\text { Classified as } \\
\text { soft plaque }\end{array}$ & 5.03 & 19.11 & 0.09 & 24.23 \\
\hline $\begin{array}{l}\text { Classified as } \\
\text { fibrous plaque }\end{array}$ & 2.43 & 67.67 & 0.44 & 70.54 \\
\hline $\begin{array}{l}\text { Classified as } \\
\text { calcium }\end{array}$ & 0.00 & 2.11 & 3.12 & 5.23 \\
\hline Totals & 7.46 & 88.89 & 3.65 & 100.00 \\
\hline
\end{tabular}


Table 2. Area comparison for different segmentations and classifications

\begin{tabular}{|l|l|l|l|}
\hline 'True' mask & Mask to compare & $\begin{array}{l}\text { Mean error soft plaque } \\
\text { (pixels) }\end{array}$ & $\begin{array}{l}\text { Mean error calcium } \\
\text { (pixels) }\end{array}$ \\
\hline Cardiologist 1 & Cardiologist 2 & $4.28 \pm 3.45$ & $1.61 \pm 0.68$ \\
\hline Cardiologist 1 & Cardiologist 3 & - & $1.14 \pm 0.65$ \\
\hline Cardiologist 1 & AdaBoost & $5.30 \pm 5.08$ & $0.71 \pm 0.21$ \\
\hline Cardiologist 1 & Snake & $5.71 \pm 5.40$ & $1.86 \pm 1.49$ \\
\hline AdaBoost & Snake & $0.78 \pm 0.50$ & $1.06 \pm 0.94$ \\
\hline
\end{tabular}

\subsection{Results of Stop \& Go Snake}

In addition to the classification map, the AdaBoost classifier also returns confidence rates and thresholds. Every confidence rate above the threshold represents the probability that the point belongs to the searched class, while a lower rate indicates that the pixel does not belong to this class. In our case, the likelihood map for soft plaque was derived from the confidence rates of the classification between fibrous plaque and calcium against soft plaque. Only the rates below the threshold were taken into account and the map was inverted before using it as a likelihood map. The likelihood map for calcium resulted from the confidence rates above the threshold for the separation of calcium and fibrous plaque.

For the actual evolution of the snake over the likelihood map, the Level Sets formulation by Osher and Sethian [14] was implemented using an explicit Euler scheme. Pujol et al. [1] found that the fastest Stop \& Go snake configuration uses $\left\{V_{0}=1.3, t=1.3\right.$, and $\left.\alpha=0.23\right\}$, while a snake fully concerned with accuracy and smoothness uses $\left\{V_{0}=0.2, t=0.5\right.$, and $\left.\alpha=0.6\right\}$. For this study, the tradeoff configuration was used, being $\left\{V_{0}=1, t=0.5\right.$, and $\left.\alpha=0.6\right\}$. Furthermore, 300 iteration steps and a $\beta$ of 150 were applied. Using those parameters, the Stop \& Go snake was applied on all images with soft plaque and calcium. After snake deformation, the plaque boundaries found by the cardiologist were superimposed on the mask found for soft plaque or calcium, in order to exclude the catheter and other artifacts. Furthermore, very small regions were omitted. The final output is mapped on the original images in Cartesian coordinates and is shown in columns D of figures 2 and 3. The white lines indicate the soft plaque found by the snake, while the black represent the calcium.

It can be seen that the selection of soft plaque is weak. However, the calcium detection is reasonable. Unfortunately, it is not trivial to analyze the snake results statistically. In this study, we propose a comparison of the areas of interest found with the snake and the same areas in the cardiologist's segmentation. We considered 20 images with soft plaque and 8 images with calcium. The error was determined as the area of the regions in the different masks that did not overlap, normalized by the total area of the 'true' mask, for example the cardiologist's map. To get more insight in intraobserver variability and AdaBoost performance as well, five different comparisons were made (see Table 2). As can be seen from the error rates in this table, it is quite difficult to detect soft plaque well, starting with the segmentation of the different cardiologists. The results for calcium are 
much better. The boosted decision stumps algorithm can be seen to perform better (more similar to the cardiologists 'ground truth') than the snake on finding the regions of interest for this tissue type.

\section{Conclusion}

In this study, various tools for pattern recognition were considered in an attempt to obtain automatic segmentation of plaque tissues. Different texture features were extracted and the AdaBoost classifier and the Stop \& Go snake both gave encouraging results segmenting calcium. These explicit and implicit classification methods both have their advantages and drawbacks. AdaBoost is a little more precise, but considers each point just within its neighborhood. Conversely, the Stop \& Go snake organizes points in groups. However, for soft plaque, this step needs some more attention in future research. Furthermore, classification results should be corrected for shadowing caused by guide wires and calcifications.

\section{References}

1. Pujol, O., Gil, D., Radeva, P.: Fundamentals of stop and go active models. Image and Vision Computing 23 (2005) 681-691

2. Nailon, W., McLaughlin, S.: Intravascular ultrasound image interpretation. Proc. of ICPR, Austria. IEEE Computer Society Press: USA (1996) 503-506

3. Zhang, X., Sonka, M.: Tissue characterization in intravascular ultrasound images. IEEE Trans. on Medical Imaging 17 (1998) 889-899

4. Pujol, O., Radeva, P.: Near real time plaque segmentation of ivus. Proc. of Computers in Cardiology (2003) 69-72

5. Pujol, O., et al.: Adaboost to classify plaque appearance in ivus images. Progress in Pattern Recognition, Image Analysis, and Appl.: LNCS 3287 (2004) 629-636

6. Roy Cardinal, M.H., et al.: Intravascular ultrasound image segmentation: A fastmarching method. MICCAI: LNCS 2879 (2003) 432-439

7. Pujol, O., Radeva, P.: Texture segmentation by statistic deformable models. Int. J. of Image and Graphics 4 (2004) 433-452

8. Roy Cardinal, M.H., et al.: Automatic 3d segmentation of intravascular ultrasound images using region and contour information. MICCAI: LNCS 3749 (2005) 319 326

9. Freund, Y., Schapire, R.: A decision-theoretic generalization of on-line learning and an application to boosting. J. of Comp. and Syst. Sciences 55 (1997) 119-139

10. Ojala, T., Pietikainen, M., Maenpaa, T.: Multiresolution gray-scale and rotation invariant texture classification with local binary patterns. IEEE Trans. on Pattern Analysis and Machine Intelligence 24 (2002) 971-987

11. Haralick, R., Shanmugam, K., Dinstein, I.: Textural features for image classification. IEEE Trans. on System, Man, Cybernetics 3 (1973) 610-621

12. $\mathrm{Qu}, \mathrm{Y}$., et al.: Boosted decision tree analysis of surface-enhanced laser desorption/ionization mass spectral serum profiles discriminates prostate cancer from non-cancer patients. Clinical Chemistry 48 (2002) 1835-1843

13. Caselles, V., Kimmel, R., Sapiro, G.: Geodesic active contours. Int. J. of Computer Vision 22 (1997) 61-79

14. Osher, S., Sethian, J.: Fronts propagating with curvature-dependent speed: algorithms based on hamilton-jacobi formulations. J. of Comput. Physics 79 (1988) 